\title{
Perturbative renormalization functions of local operators for staggered fermions with stout improvement
}

\author{
M. Constantinou, M. Costa and H. Panagopoulos* \\ Department of Physics, University of Cyprus, \\ POB 20537, 1678 Nicosia, Cyprus
}

\begin{abstract}
In this paper we present the perturbative computation of the renormalization functions for the quark field and for a complete set of ultralocal fermion bilinears. The computation of the relevant Green's functions are carried out at one-loop level for the staggered action using massive fermions. The gluon links which appear both in the fermion action and in the definition of the bilinears are improved by applying a stout smearing procedure up to two times, iteratively. In the gluon sector we employ the Symanzik improved gauge action for different sets of values of the Symanzik coefficients. The renormalization functions are presented in (two variants of) the RI' and in the $\overline{\mathrm{MS}}$ renormalization scheme; the dependence on all stout parameters, as well as on the fermion mass, the gauge fixing parameter and the renormalization scale, is shown explicitly. This work is related to our recent paper [Phys. Rev. D 86, 094512 (2012)]. To make our results easily accessible to the reader, we include them in the distribution package of this paper as a Mathematica input file, "Staggered.m".

PACS numbers: 11.15.Ha, 12.38.Gc, 12.38.Aw, 12.38.-t
\end{abstract}

\section{INTRODUCTION}

In recent years, significant improvements have been made in the use of matrix elements of operators made out of quark fields to extract mass spectra, decay constants, and a plethora of hadronic properties [1-3]. Although naive (unimproved) staggered fermions were introduced more than three decades ago [4], their discretization errors and their relatively large taste mixing posed a limit on the accuracy of results from simulations, despite their relatively low computational cost. This situation called for improvement; the outcome of such efforts was some of the most accurate discretizations used to date for high-precision simulations. One specific direction regards improving the fermion action (see, e.g. $[5,6]$ ); in particular, the introduction of stout links in the action which has recently been put to use $[7,8]$ allows simulations to be carried out at near physical parameters. Compared to most other improved formulations of staggered fermions, the above action, as well as the HISQ action, lead to smaller taste violating effects [9-11].

Changes in the lattice action and in the discretization of operators imply that renormalization functions must be determined afresh, either perturbatively or nonperturbatively. In many cases nonperturbative estimates of renormalization functions are very difficult to obtain, due to complications such as possible mixing with operators of equal or lower dimension, whose signals are hard to disentangle. For this reason, and in order to provide cross-estimates which have a reduced systematic error, the perturbative study of a variety of fermion operators is widely employed in numerical simulations of QCD on the lattice (see, e.g. [12] and references therein, also [13-17]).

Within the staggered formulation using massive fermions we compute the fermion propagator and Green's functions of a set of local taste-singlet bilinears $\mathcal{O}$ [scalar $(\mathrm{S})$, pseudoscalar $(\mathrm{P})$, vector $(\mathrm{V})$, axial $(\mathrm{A})$, and tensor $(\mathrm{T})]$. Our computation is performed to one loop and to lowest order in the lattice spacing, $a$. We also extract from the above the renormalization functions (RFs) of the quark field $Z_{q}$, quark mass $Z_{m}$, and fermion bilinears $Z_{\mathcal{O}}$. This work is a continuation to our recent paper [8], in which we presented our perturbative results for $Z_{q}, Z_{\mathrm{T}}$, and $Z_{\mathrm{S}}$; it is the first one-loop computation of these quantities using staggered fermions with stout links. In the present paper, we provide the details of the perturbative calculation and our results for the propagator and for the Green's functions, as well as the renormalization functions of all operators, including the vector, axial, and pseudoscalar cases. Older results with staggered fermions [13] in the absence of stout smearing and for the Wilson gluon action are in complete agreement with our results; perturbartive results related to alternative improvements of the staggered action can be found, e.g., in Refs. $[18,19]$.

Stout links [20] rather than ordinary links have been used both in the fermion action and in bilinear operators. Following Ref. [8], we use two steps of stout smearing with generic smearing parameters $\left(\omega_{1}, \omega_{2}\right)$. We emphasize

*Electronic address: marthac@ucy.ac.cy, kosta.marios@ucy.ac.cy, haris@ucy.ac.cy 
that the results for the bilinear Green's functions depend on four stout parameters, two due to the action smearing $\left(\omega_{A_{1}}, \omega_{A_{2}}\right)$ and two more coming from the smearing of the operator $\left(\omega_{\mathcal{O}_{1}}, \omega_{\mathcal{O}_{2}}\right)$; no numerical value needs to be specified for these parameters. The extension to further steps of stout smearing can be achieved with relative ease. For gluons we employ the Symanzik improved action. Our final expressions for the Green's functions exhibit a rather nontrivial dependence on the external momentum $(q)$ and the fermion mass $(m)$, and they are polynomial functions of the gauge parameter $(\alpha)$, stout parameters $\left(\omega_{A_{i}}, \omega_{\mathcal{O}_{i}}\right)$, and coupling constant $(g)$; furthermore, most numerical coefficients in these expressions depend on the Symanzik parameters of the gluon action.

The one-loop expressions for the renormalization functions are presented in the mass-independent RI' scheme; for the vector and axial renormalization functions we also employ an alternative RI' scheme which might be more useful in renormalizing nonperturbative matrix elements. Furthermore, for comparison with experimental determinations and phenomenological estimates, it is useful to present our results also in the $\overline{\mathrm{MS}}$ scheme; we do so, paying particular attention to the possible alternative definitions of $\gamma_{5}$.

Results for $Z_{\mathrm{q}}, Z_{\mathrm{m}}, Z_{\mathcal{O}}$ exist for simpler actions to $\mathcal{O}\left(g^{4}\right)$ and/or $\mathcal{O}\left(g^{2} a^{n}\right)$, see e.g., Refs. [16, 21] for two-loop renormalization of flavor singlet and nonsinglet local fermion bilinears, Ref. [15] for $Z_{\mathrm{m}}$ to two loops, Ref. [22] for oneloop renormalization of the fermion propagator and bilinears to $\mathcal{O}\left(a^{1}\right)$, and Refs. [17, 23, 24] for the fermion propagator and bilinears with 0 and 1 derivatives to one-loop and to $\mathcal{O}\left(a^{2}\right)$. The extension of the present computation beyond one loop and/or beyond $\mathcal{O}\left(a^{0}\right)$ becomes exceedingly complicated: One reason for this is the appearance of divergences in nontrivial corners of the Brillouin zone; also, a two-loop calculation requires vertices with up to four gluons, which are extremely lengthy in the presence of stout links (estimated length: $>10^{6}$ terms).

To make our results easily accessible, we accompany this paper with an electronic document in the form of a Mathematica input file ("Staggered.m"), allowing the reader to extract the expressions for many choices of the action parameters. This document contains the one-loop inverse fermion propagator, the one-loop amputated Green's functions relevant to all ultralocal operators, and the renormalization functions in the RI' scheme for the fermion field and for all bilinears. In addition, in Staggered.m we provide the expressions for the one- and two-gluon "doubly stout" links for different stout parameters in the first and second smearing step.

The outline of this paper is as follows: Sec. II regards a brief theoretical background in which we introduce the formulation of the action and of the operators which we employ. Section III contains a summary of the calculational procedure for the fermion propagator and for the Green's functions of the bilinear operators. We also present the most general results for these quantities using the tree-level Symanzik improved gluon action. The renormalization functions are derived in Sec. IV for different renormalization schemes, and we provide their expressions for tree-level Symanzik gluons. Finally, we conclude in Sec. V with a discussion of our results and possible future extensions of our work. For completeness, we have included 3 Appendices containing: A: the stout smeared links, B: the numerical results of the propagator for the Wilson and tree-level Symanzik actions, and C: a description of the Mathematica file "Staggered.m".

\section{FORMULATION}

\section{A. Lattice actions}

Our perturbative calculation makes use of the staggered fermion action. Let us briefly go over the derivation of the latter, starting from the naive fermion action

$$
S^{F}=a^{4} \sum_{x, f, \mu} \bar{\psi}^{f}(x)\left(\gamma_{\mu} D_{\mu}\right) \psi^{f}(x)+a^{4} \sum_{x, f} m_{f} \bar{\psi}^{f}(x) \psi^{f}(x),
$$

where $f$ is a flavor index. Given that, to one loop in perturbation theory, the quantities of interest do not depend on the number of flavors, we will drop the index $f$ from this point on. The covariant derivative $D_{\mu}$ is defined as

$$
D_{\mu} \psi(x)=\frac{1}{2 a}\left[U_{\mu}(x) \psi(x+a \hat{\mu})-U_{\mu}^{\dagger}(x-a \hat{\mu}) \psi(x-a \hat{\mu})\right] .
$$

The absence of the Wilson term in the naive action of Eq. (1) leads to the well-known doubling problem. The standard passage to the staggered action entails the following change of basis:

$$
\begin{array}{r}
\psi(x)=\gamma_{x} \chi(x), \quad \bar{\psi}(x)=\bar{\chi}(x) \gamma_{x}^{\dagger}, \\
\gamma_{x}=\gamma_{1}^{n_{1}} \gamma_{2}^{n_{2}} \gamma_{3}^{n_{3}} \gamma_{4}^{n_{4}}, \quad x=\left(a n_{1}, a n_{2}, a n_{3}, a n_{4}\right), \quad n_{i} \in \mathbb{Z} .
\end{array}
$$


Using the equalities

$$
\gamma_{\mu} \gamma_{x}=\eta_{\mu}(x) \gamma_{x+a \hat{\mu}} \quad \text { and } \quad \gamma_{x}^{\dagger} \gamma_{x}=\mathbb{1}, \quad \eta_{\mu}(x)=(-1)^{\sum_{\nu<\mu} n_{\nu}},
$$

the lattice fermion action takes the form

$$
S^{F}=a^{4} \sum_{x} \sum_{\mu} \frac{1}{2 a} \bar{\chi}(x) \eta_{\mu}(x)\left[U_{\mu}(x) \chi(x+a \hat{\mu})-U_{\mu}^{\dagger}(x-a \hat{\mu}) \chi(x-a \hat{\mu})\right]+a^{4} \sum_{x} m \bar{\chi}(x) \chi(x) .
$$

Thus far, we have rewritten the usual lattice action. But the crucial step now is that the Dirac matrices have disappeared, and they have been replaced by the phase factors $\eta_{\mu}(x)$; in the new basis, the naive action consists of four identical parts, one for each value of the spinor index carried by the spinor $\chi$. Dropping this index altogether leads to the standard staggered fermion action $S_{\text {stag }}$ containing four rather than 16 fermion "tastes" (doublers). These four tastes contain a total of 16 components, which are split over a unit hypercube by assigning only a single fermion field component to each lattice site.

Following the nonperturbative work of Ref. [8] we apply stout smearing according to Ref. [20] to all links appearing in $S_{\text {stag }}$ : Each link $U_{\mu}(x)=\exp \left(i g a A_{\mu}(x+a \hat{\mu} / 2)\right)$ is replaced by a stout link $\widetilde{U}_{\mu}(x)$ defined as [25]

$$
\widetilde{U}_{\mu}(x)=e^{i Q_{\mu}(x)} U_{\mu}(x)
$$

where the definition of $Q_{\mu}(x)$ is

$$
Q_{\mu}(x)=\frac{\omega}{2 i}\left[V_{\mu}(x) U_{\mu}^{\dagger}(x)-U_{\mu}(x) V_{\mu}^{\dagger}(x)-\frac{1}{3} \operatorname{Tr}\left(V_{\mu}(x) U_{\mu}^{\dagger}(x)-U_{\mu}(x) V_{\mu}^{\dagger}(x)\right)\right] .
$$

$\omega$ is a tunable parameter, called a stout smearing parameter, and $V_{\mu}(x)$ represents the sum over all staples associated with the link, $U_{\mu}(x)$. In the present work we need the contributions of $Q_{\mu}(x)$ up to two gluons, to which the trace terms in Eq. (7) are irrelevant; the contributions can be read from the terms

$$
Q_{\mu}^{\text {up to } 2-\text { gluons }}(x)=\frac{\omega}{2 i} \sum_{\rho= \pm 1}^{ \pm 4}\left(U_{\rho}(x) U_{\mu}(x+a \hat{\rho}) U_{\rho}^{\dagger}(x+a \hat{\mu}) U_{\mu}^{\dagger}(x)-U_{\mu}(x) U_{\rho}(x+a \hat{\mu}) U_{\mu}^{\dagger}(x+a \hat{\rho}) U_{\rho}^{\dagger}(x)\right)
$$

$\left(U_{-\rho}(y) \equiv U_{\rho}^{\dagger}(y-a \hat{\rho}), \rho>0\right)$. The above procedure can be performed iteratively by dressing the links more than once in order to improve the convergence to the continuum limit. In the framework of our calculation we use "doubly stout" links

$$
\widetilde{\widetilde{U}}_{\mu}(x)=e^{i \widetilde{Q}_{\mu}(x)} \widetilde{U}_{\mu}(x),
$$

where $\widetilde{Q}$ is defined as in Eq. (7) but using $\widetilde{U}$ as links (also in the construction of $V_{\mu}$ ). Such links have been employed in numerical simulations in Refs. [7, 10]. To obtain results that are as general as possible, we use different stout parameters, $\omega$, in the first $\left(\omega_{1}\right)$ and the second $\left(\omega_{2}\right)$ smearing iteration. This allows for further optimization of improvement, by separate tuning of the two parameters; it also provides a check of the perturbative calculation by comparing the limit $\omega_{1}=0$ (or $\omega_{2}=0$ ) to the case of a single step of stout smearing. We smear both the links in $S_{\text {stag }}$ and those in bilinear operators (see following subsection), so that we have a total of four stout parameters that we keep different from one another. In Appendix A we present the one-gluon link, $U^{(1)}$, for general $\omega_{1}$ and $\omega_{2}$, as well as the two-gluon link, $U^{(2)}$; due to space limitations, the lengthy expression for $U^{(2)}$ (a total of $\sim 500$ terms) has been presented only for $\omega_{2}=0$. The general expression for the two-gluon link with $0 \neq \omega_{1} \neq \omega_{2} \neq 0$ is provided in the Mathematica file.

For gluons we employ the Symanzik improved action, involving Wilson loops with four and six links $(1 \times 1$ plaquette (a), $1 \times 2$ rectangle (b), $1 \times 2$ chair $(\mathrm{c})$, and $1 \times 1 \times 1$ parallelogram (d) wrapped around an elementary $3 \mathrm{D}$ cube), as shown in Fig. 1:

$$
\begin{aligned}
S_{G}=\frac{2}{g_{0}^{2}} & {\left[c_{0} \sum_{\text {plaq. }} \operatorname{Re} \operatorname{Tr}\left\{1-U_{\text {plaq. }}\right\}+c_{1} \sum_{\text {rect. }} \operatorname{Re} \operatorname{Tr}\left\{1-U_{\text {rect. }}\right\}\right.} \\
& \left.+c_{2} \sum_{\text {chair }} \operatorname{Re} \operatorname{Tr}\left\{1-U_{\text {chair }}\right\}+c_{3} \sum_{\text {paral. }} \operatorname{Re} \operatorname{Tr}\left\{1-U_{\text {paral. }}\right\}\right] .
\end{aligned}
$$




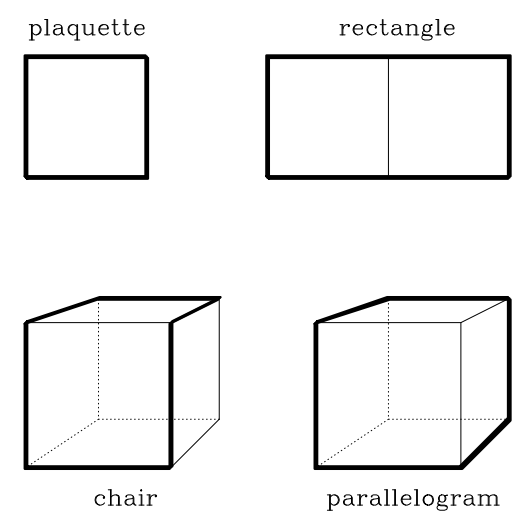

FIG. 1: The four Wilson loops of the gluon action.

The coefficients $c_{i}$ can in principle be chosen arbitrarily, subject to the following normalization condition, which ensures the correct classical continuum limit of the action

$$
c_{0}+8 c_{1}+16 c_{2}+8 c_{3}=1 .
$$

Some popular choices of values for $c_{i}$ used in numerical simulations will be considered in this work and are itemized in Table I of Ref. [24]. They include the Wilson case $\left(c_{0}=1, c_{1}=c_{2}=c_{3}=0\right)$, and the tree-level Symanzik, TILW (tadpole improved Lüscher-Weisz), Iwasaki, and DBW2 (doubly blocked Wilson action) actions. In the results presented in paper form we use the tree-level Symanzik action $\left(c_{0}=5 / 3, c_{1}=-1 / 12, c_{2}=c_{3}=0\right)$. Our one-loop Feynman diagrams do not involve pure gluon vertices, and the gluon propagator depends only on three combinations of the Symanzik parameters:

$$
c_{0}+8 c_{1}+16 c_{2}+8 c_{3}=1, \quad c_{2}+c_{3}, \quad c_{1}-c_{2}-c_{3} .
$$

Therefore, with no loss of generality we choose $c_{2}=0$.

\section{B. Definition of operators}

In the staggered formalism one defines fields that live on the corners of four-dimensional elementary hypercubes of the lattice $[13,26,27]$. The position of a hypercube inside the lattice is denoted by the index $y$, where $y$ is a four-vector with components $y_{\mu}$, which are even integers $\left(y_{\mu} \in 2 \mathbb{Z}\right)$. The position of a fermion field component within a specific hypercube is defined by one additional four-vector index, $C\left(C_{\mu} \in\{0,1\}\right)$.

To be able to obtain the correct continuum limit, both for the action and for operators containing fermions, we relate $\chi$ with the physical field $Q_{\beta, b}$ ( $\beta$ : Dirac index, $b$ : taste index). In standard notation:

$$
\chi(y)_{C} \equiv \chi(a y+a C) / 4=\sum_{\beta, b}\left(\frac{1}{2} \xi_{C}\right)_{\beta, b} Q_{\beta, b}(y), \quad Q_{\beta, b}(y) \equiv \frac{1}{2} \sum_{C}\left(\gamma_{C}\right)_{\beta, b} \chi(y)_{C},
$$

where $\xi_{C}$ is defined similarly to $\gamma_{C}$ [Eq. (3)], that is $\xi_{C}=\xi_{1}^{C_{1}} \xi_{2}^{C_{2}} \xi_{3}^{C_{3}} \xi_{4}^{C_{4}}, \quad \xi_{\mu}=\left(\gamma_{\mu}^{\star}\right)$. In terms of the field $Q$ one can now define fermion bilinear operators as follows:

$$
\mathcal{O}_{\Gamma, \xi}=\bar{Q}(\Gamma \otimes \xi) Q
$$

where $\Gamma$ and $\xi$ are arbitrary $4 \times 4$ matrices acting on the Dirac and taste indices of $Q_{\beta, b}$, respectively. After rotating into the staggered basis, the operator $\mathcal{O}_{\Gamma, \xi}$ can be written as [13]

$$
\begin{array}{r}
\mathcal{O}_{\Gamma, \xi}=\sum_{C, D} \bar{\chi}(y)_{C}(\overline{\Gamma \otimes \xi})_{C D} \chi(y)_{D}, \\
(\overline{\Gamma \otimes \xi})_{C D} \equiv \frac{1}{4} \operatorname{Tr}\left[\gamma_{C}^{\dagger} \Gamma \gamma_{D} \xi\right] .
\end{array}
$$


In this work we focus on taste-singlet operators, thus $\xi=\mathbb{1}$.

The operator of Eq. (14) is clearly not gauge invariant, since $\bar{\chi}$ and $\chi$ are defined at different points of the hypercube. To restore gauge invariance, we insert the average of products of gauge link variables along all possible shortest paths connecting the sites $y+C$ and $y+D$. This average is denoted by $U_{C, D}$ and the gauge invariant-operator is now

$$
\mathcal{O}_{\Gamma} \equiv \mathcal{O}_{\Gamma, \mathbb{1}}=\sum_{C, D} \bar{\chi}(y)_{C}(\overline{\Gamma \otimes \mathbb{1}})_{C D} U_{C, D} \chi(y)_{D}
$$

From the definition of Eq. (15), as well as the equalities of Eq. (4), we can further simplify the expression for the operator $\mathcal{O}_{\Gamma}$, using

$$
\begin{aligned}
\frac{1}{4} \operatorname{Tr}\left[\gamma_{C}^{\dagger} \mathbb{1} \gamma_{D}\right] & =\delta_{C, D}, \\
\frac{1}{4} \operatorname{Tr}\left[\gamma_{C}^{\dagger} \gamma_{\mu} \gamma_{D}\right] & =\delta_{C, D+\hat{\mu}} \eta_{\mu}(D) \\
\frac{1}{4} \operatorname{Tr}\left[\gamma_{C}^{\dagger} \sigma_{\mu \nu} \gamma_{D}\right] & =\frac{1}{i} \delta_{C, D+\hat{\mu}+\hat{\nu}} \eta_{\nu}(D) \eta_{\mu}(D+\hat{\nu}), \\
\frac{1}{4} \operatorname{Tr}\left[\gamma_{C}^{\dagger} \gamma_{5} \gamma_{\mu} \gamma_{D}\right] & =\delta_{C, D+\hat{\mu}+(1,1,1,1)} \eta_{\mu}(D) \eta_{1}(D+\hat{\mu}) \eta_{2}(D+\hat{\mu}) \eta_{3}(D+\hat{\mu}) \eta_{4}(D+\hat{\mu}), \\
\frac{1}{4} \operatorname{Tr}\left[\gamma_{C}^{\dagger} \gamma_{5} \gamma_{D}\right] & =\delta_{C, D+(1,1,1,1)} \eta_{1}(D) \eta_{2}(D) \eta_{3}(D) \eta_{4}(D),
\end{aligned}
$$

where $\sigma_{\mu \nu}=\left[\gamma_{\mu}, \gamma_{\nu}\right] /(2 i)$. Here and below, in expressions such as $D+\hat{\mu}$, the sum is to be taken modulo 2 . Using Eq. (17), the operators can be written as

$$
\begin{aligned}
\mathcal{O}_{S}(y) & =\sum_{D} \bar{\chi}(y)_{D} \chi(y)_{D} \\
\mathcal{O}_{V}(y) & =\sum_{D} \bar{\chi}(y)_{D+\hat{\mu}} U_{D+\hat{\mu}, D} \chi(y)_{D} \eta_{\mu}(D) \\
\mathcal{O}_{T}(y) & =\frac{1}{i} \sum_{D} \bar{\chi}(y)_{D+\hat{\mu}+\hat{\nu}} U_{D+\hat{\mu}+\hat{\nu}, D} \chi(y)_{D} \eta_{\nu}(D) \eta_{\mu}(D+\hat{\nu}) \\
\mathcal{O}_{A}(y) & =\sum_{D} \bar{\chi}(y)_{D+\hat{\mu}+(1,1,1,1)} U_{D+\hat{\mu}+(1,1,1,1), D} \chi(y)_{D} \eta_{\mu}(D) \eta_{1}(D+\hat{\mu}) \eta_{2}(D+\hat{\mu}) \eta_{3}(D+\hat{\mu}) \eta_{4}(D+\hat{\mu}) \\
\mathcal{O}_{P}(y) & =\sum_{D} \bar{\chi}(y)_{D+(1,1,1,1)} U_{D+(1,1,1,1), D} \chi(y)_{D} \eta_{1}(D) \eta_{2}(D) \eta_{3}(D) \eta_{4}(D)
\end{aligned}
$$

With the exception of the scalar operator, the remaining operators contain averages of products of up to four links (in orthogonal directions) between the fermion and the antifermion fields. For example, the average entering the tensor operator of Eq. (20) is

$$
U_{D+\hat{\mu}+\hat{\nu}, D}=\frac{1}{2}\left[\tilde{\tilde{U}}_{\nu}^{\dagger}(a y+a D+a \hat{\mu}) \tilde{\tilde{U}}_{\mu}^{\dagger}(a y+a D)+\{\mu \leftrightarrow \nu\}\right],
$$

valid when $(D+\hat{\mu}+\hat{\nu})_{i} \geq D_{i}, i=1,2,3,4$, and similarly for all other cases.

\section{CALCULATION OF GREEN'S FUNCTIONS}

In this section we describe some of the technical aspects of the calculation and present our results for one-loop Green's functions. As a starting point one must derive the vertices for the staggered action and the operators, up to two gluons, as required in our one-loop computation. For this reason one may use an equivalent expression of $\eta_{\mu}(x)$ appearing in the action

$$
\eta_{\mu}(x)=e^{i \pi \bar{\mu} n}, \quad x=a n, \quad \bar{\mu}=\sum_{\nu=1}^{\mu-1} \hat{\nu} .
$$

Using this form of $\eta_{\mu}(x)$, instead of the definition of Eq. (4), simplifies the expression for $\mathcal{O}_{\Gamma}$ in terms of Fourier transformed fields, $\tilde{\chi}(k), \tilde{A}_{\rho}(k) \equiv \tilde{A}_{\rho}^{c}(k) T^{c}$ : 


$$
\begin{aligned}
\mathcal{O}_{\Gamma} & =\int_{-\pi}^{\pi} \frac{d^{4} k_{1}}{(2 \pi)^{4}} \int_{-\pi}^{\pi} \frac{d^{4} k_{2}}{(2 \pi)^{4}} \tilde{\bar{\chi}}\left(k_{1}\right) V_{\Gamma}\left(k_{1}, k_{2}\right) \tilde{\chi}\left(k_{2}\right) \\
& +\sum_{c, \rho} \int_{-\pi}^{\pi} \frac{d^{4} k_{1}}{(2 \pi)^{4}} \int_{-\pi}^{\pi} \frac{d^{4} k_{2}}{(2 \pi)^{4}} \int_{-\pi}^{\pi} \frac{d^{4} k_{3}}{(2 \pi)^{4}} \tilde{\bar{\chi}}\left(k_{1}\right) V_{\Gamma}^{c, \rho}\left(k_{1}, k_{2}, k_{3} ; \omega_{1}, \omega_{2}\right) \tilde{\chi}\left(k_{2}\right) \tilde{A}_{\rho}^{c}\left(k_{3}\right) \\
& +2-\text { gluon terms }+\cdots
\end{aligned}
$$

Thus, after Fourier transformation, the quark-antiquark vertices of Eqs. (18) - (20), without stout smearing, become

$$
\begin{aligned}
& V_{S}\left(k_{1}, k_{2}\right)=\delta\left(k_{2}-k_{1}\right) \\
& V_{V}\left(k_{1}, k_{2}\right)=\delta\left(k_{2}-k_{1}+\pi \bar{\mu}\right) e^{-i k_{1} \mu} \\
& V_{T}\left(k_{1}, k_{2}\right)=\delta\left(k_{2}-k_{1}+\pi \bar{\mu}+\pi \bar{\nu}\right) e^{-i k_{1} \mu} e^{-i k_{1} \nu}(\nu>\mu), \\
& V_{A}\left(k_{1}, k_{2}\right)=\eta_{\mu}(\bar{\mu}) \delta\left(k_{2}-k_{1}+\pi \sum_{\nu=1}^{4} \bar{\nu}+\pi \bar{\mu}\right) e^{-i\left(k_{1_{1}}+k_{1_{2}}+k_{1_{3}}+k_{1_{4}}-k_{1_{\mu}}\right)}, \\
& V_{P}\left(k_{1}, k_{2}\right)=\delta\left(k_{2}-k_{1}+\pi \sum_{\nu=1}^{4} \bar{\nu}\right) e^{-i\left(k_{1_{1}}+k_{1_{2}}+k_{1_{3}}+k_{1_{4}}\right)} .
\end{aligned}
$$

As for vertices containing gluons, we give here as an example the one-gluon vertex of the vector operator, including double stout smearing:

$$
\begin{aligned}
V_{V}^{c, \rho}\left(k_{1}, k_{2}, k_{3} ; \omega_{1}, \omega_{2}\right)= & i g T^{c}\left[\cos \left(\frac{k_{3 \mu}}{2}+k_{1 \mu}\right) \delta\left(k_{3}-k_{2}+k_{1}+\pi \bar{\mu}+\pi \mu\right)+i \delta\left(k_{3}-k_{2}+k_{1}+\pi \bar{\mu}\right) \sin \left(\frac{k_{3 \mu}}{2}+k_{1 \mu}\right)\right] \\
& \cdot\left\{4 \sin \left(\frac{k_{3 \rho}}{2}\right) \sin \left(\frac{k_{3 \mu}}{2}\right)\left(\omega_{1}+\omega_{2}+2 \omega_{1} \omega_{2}\left(-4+\sum_{\sigma} \cos \left(k_{1 \sigma}\right)\right)\right)\right. \\
+ & \left.\delta_{\rho \mu}\left(\left(8 \omega_{1}-1\right)\left(8 \omega_{2}-1\right)+2 \sum_{\sigma} \cos \left(k_{3 \sigma}\right)\left(\omega_{1}+\omega_{2}+2 \omega_{1} \omega_{2}\left(-8+\sum_{\tau} \cos \left(k_{3 \tau}\right)\right)\right)\right)\right\},(31)
\end{aligned}
$$

where $\mu$ is the index of the inserted Dirac matrix $\left(\gamma_{\mu}\right)$ and $\rho$ is the index of the gluon.

Given that the argument $y$ of the operators $\mathcal{O}_{\Gamma}$ runs only over even integers, summation over the position of $\mathcal{O}_{\Gamma}$, followed by Fourier transformation leads to expressions of the form

$$
\sum_{y_{\mu} \in 2 \mathbb{Z}} e^{i y \cdot k}=\frac{1}{16}(2 \pi)^{4} \sum_{C} \delta_{2 \pi}(k+\pi C)
$$

where $\delta_{2 \pi}(k)$ stands for the standard periodic $\delta$ function with nonvanishing support at $k \bmod 2 \pi=0$. Since contributions to the continuum limit come from the neighborhood of each of the 16 poles of the external momenta $q$, at $q_{\mu}=(\pi / a) C_{\mu}$, it is useful to define $q_{\mu}^{\prime}$ and $C_{\mu}$ through

$$
q_{\mu}=q_{\mu}^{\prime}+\frac{\pi}{a} C_{\mu} \quad\left(\bmod \left(\frac{2 \pi}{a}\right)\right), \quad\left(C_{\mu} \in\{0,1\}\right),
$$

where the "small" (physical) part $q^{\prime}$ has each of its components restricted to one-half of the Brillouin zone: $-\pi /(2 a) \leq$ $q_{\mu}^{\prime} \leq \pi /(2 a)$. Thus, conservation of external momenta takes the form

$$
\delta_{2 \pi}\left(a q_{1}-a q_{2}+\pi D\right)=\frac{1}{a} \delta\left(q_{1}^{\prime}-q_{2}^{\prime}\right) \prod_{\mu} \delta_{C_{1 \mu}-C_{2 \mu}+D_{\mu}, 0} .
$$

For the algebraic operations involved in evaluating the Feynman diagrams relevant to this calculation, we make use of our symbolic package in Mathematica; a description of this can be found, e.g., in a previous publication [23]. 


\section{A. Fermion propagator}

We compute the one-loop correction to the fermion propagator in order to obtain the renormalization function of the fermion field, an essential ingredient for the renormalization of the operators $\mathcal{O}_{\Gamma}$. The tree-level fermion propagator in the basis of the $\chi$ fields can be written as

$$
S_{\text {tree }}\left(q_{1}, q_{2}\right)=(2 \pi)^{4} \frac{-\frac{i}{a} \sum_{\mu} \sin \left(a q_{1 \mu}\right) \delta\left(q_{1}-q_{2}+\frac{\pi \bar{\mu}}{a}\right)+m \delta\left(q_{1}-q_{2}\right)}{\frac{1}{a^{2}} \sum_{\mu} \sin ^{2}\left(a q_{1 \mu}\right)+m^{2}} .
$$

The one-loop Feynman diagrams that enter the calculation of the two-point, one-particle irreducible (1PI), amputated Green's function $S^{-1}(p)$ are illustrated in Fig. 2.
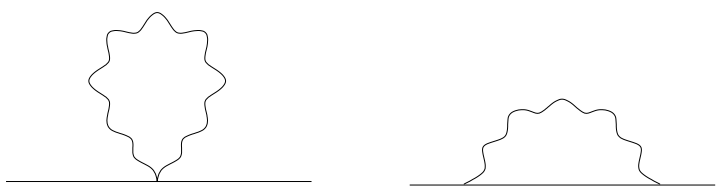

FIG. 2: One-loop diagrams contributing to the fermion propagator. Wavy (solid) lines represent gluons (fermions).

We have computed $S^{-1}(p)$ for general values of the gauge parameter $\alpha(\alpha=0$ : Landau gauge, $\alpha=1$ : Feynman gauge), the stout smearing parameters $\omega_{A_{1}}, \omega_{A_{2}}$, the Lagrangian mass $m$, the number of colors $N_{c}$, and the external momenta $q_{1}, q_{2}$. We have obtained results using different sets of values for the Symanzik coefficients (shown in Ref. [23]). In presenting our result, Eq. (36), for $S^{-1}(p)$ up to one loop, the quantities $e_{1}, e_{2}$ are numerical coefficients that depend on the Symanzik coefficients and the stout smearing parameters. In Appendix B we provide the general form of $e_{1}, e_{2}$ and tabulate their numerical values for the Wilson and tree-level Symanzik cases; for other actions see Appendix C. In all expressions the systematic errors (coming from an extrapolation to infinite lattice size of our numerical loop integrals) are smaller than the last digit we present.

$$
\begin{aligned}
S_{1-\text { loop }}^{-1}= & \left(\sum_{\rho} \delta\left(q_{1}-q_{2}+\frac{\pi}{a} \bar{\rho}\right) i p_{\rho}(-1)^{C_{1 \rho}}\right) \times \\
& \quad\left[1+\frac{g^{2} C_{F}}{16 \pi^{2}}\left[\left(e_{1}+4.79201 \alpha-\alpha\left(\log \left(a^{2} m^{2}+a^{2} p^{2}\right)+\frac{m^{2}}{p^{2}}-\frac{m^{4}}{p^{4}} \log \left(1+\frac{p^{2}}{m^{2}}\right)\right)\right]\right]\right. \\
+ & \delta\left(q_{1}-q_{2}\right) m \times \\
& \quad\left[1+\frac{g^{2} C_{F}}{16 \pi^{2}}\left[e_{2}+5.79201 \alpha-(3+\alpha)\left(\log \left(a^{2} m^{2}+a^{2} p^{2}\right)+\frac{m^{2}}{p^{2}} \log \left(1+\frac{p^{2}}{m^{2}}\right)\right)\right]\right] \\
+ & \mathcal{O}\left(a^{1}\right),
\end{aligned}
$$

$q_{1}, q_{2}$ : external momenta, $\quad C_{F} \equiv \frac{N_{c}^{2}-1}{2 N_{c}}, \quad a p_{\rho} \equiv\left(a q_{1 \rho}+\frac{\pi}{2}\right)_{\bmod \pi}-\frac{\pi}{2}=\left(a q_{2 \rho}+\frac{\pi}{2}\right)_{\bmod \pi}-\frac{\pi}{2}$, and $C_{1}$ is defined in Eq. (33). Equation (36) does have the expected structure of an inverse propagator, once one identifies, in the continuum limit:

$$
\sum_{\rho} \delta\left(q_{1}-q_{2}+\frac{\pi}{a} \bar{\rho}\right) p_{\rho}(-1)^{C_{1 \rho}} \underset{a \rightarrow 0}{\longrightarrow} \delta\left(q_{1}^{\prime}-q_{2}^{\prime}\right) q_{1}^{\prime}
$$

We denote the expression in square brackets in the last line of Eq. (36) as $\Sigma_{m}\left(q^{2}, m\right)$; from this we will extract the multiplicative renormalization of the Lagrangian mass, $Z_{\mathrm{m}}$. 


\section{B. Fermion bilinears}

In the context of this work we also study the 1PI, amputated, two-point Green's functions of the operators $\mathcal{O}_{\Gamma}$, defined in Eqs. (18)-(20) up to one loop: $\Lambda_{\mathcal{O}_{\Gamma}}^{1-\text { loop }}$. The 1PI Feynman diagrams that enter the calculation of the above Green's functions are shown in Fig. 3, and include up to two-gluon vertices extracted from the operator (the cross in the diagrams). The appearance of gluon lines on the operator stems from the product $U_{C, D}$ in the operator definition $[\mathrm{Eq} \cdot(16)]^{1}$.
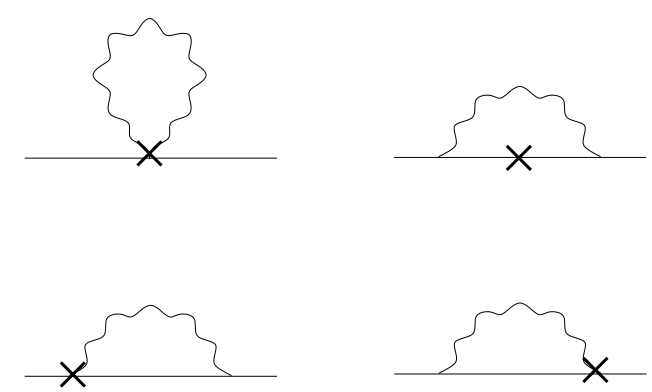

FIG. 3: One-loop diagrams contributing to the fermion-antifermion Green's functions of the bilinear operators. A wavy (solid) line represents gluons (fermions). A cross denotes an insertion of the operator $\mathcal{O}_{\Gamma}$.

Analogous expressions to Eq. (36) arise for the bilinears as well. We note that the extraction of $Z_{\mathcal{O}_{\Gamma}}$ in a massindependent scheme, such as $\mathrm{RI}^{\prime}$-MOM, necessitates evaluation of $\Lambda_{\mathcal{O}_{\Gamma}}^{1-\text { loop }}$ for $m=0$ only. Nevertheless, we have included a nonzero Lagrangian mass to our computations; this allows us to derive the renormalized Green's functions at $m \neq 0$. Comparing the latter with results using a different regularization scheme (e.g. dimensional regularization) provides another check in our computation.

Although computing the diagrams of Fig. 3 does not use the expression of the propagator [Eq. (36)], all our results shown in Eqs. (38) - (42) are expressed in terms of $e_{1}$ [see Eqs. (36) and (B1)]. The reason for that is to show explicitly the contribution of the quantities $\lambda_{\mathcal{O}}$ [Eqs. (57) - (61)] which appear in the renormalization functions $Z_{\mathcal{O}}$ [Eqs. (43) - (47)].

Dropping an overall Dirac $\delta$ function of momentum conservation, and denoting the physical momentum of the

\footnotetext{
${ }^{1}$ For $\mathcal{O}_{S}$ only the top right diagram of Fig. 3 contributes, since $U_{C, D}=\mathbb{1}$.
} 
fermion and antifermion by $p$, we obtain $\Lambda_{\mathcal{O}}^{1-l o o p}$

$$
\begin{aligned}
& \Lambda_{S}^{1-\text { loop }}=\mathbb{1}+\frac{g^{2} C_{F}}{16 \pi^{2}}\left[e_{1}-\lambda_{S}+5.79201 \alpha+i \not \supset\left(4 \alpha \frac{m^{3}}{\left(p^{2}\right)^{2}} \log \left(1+\frac{p^{2}}{m^{2}}\right)-4 \alpha \frac{m}{p^{2}}\right)\right. \\
& \left.-(\alpha+3)\left(3 \frac{m^{2}}{p^{2}} \log \left(1+\frac{p^{2}}{m^{2}}\right)+\log \left(a^{2} m^{2}+a^{2} p^{2}\right)\right)\right] \\
& \Lambda_{V}^{1-\text { loop }}=\gamma_{\mu}+\frac{g^{2} C_{F}}{16 \pi^{2}}\left[\gamma_{\mu}\left(e_{1}-\lambda_{V}+4.79201 \alpha-\alpha \frac{m^{2}}{p^{2}}-\alpha \log \left(a^{2} m^{2}+a^{2} p^{2}\right)+\alpha \frac{m^{4}}{\left(p^{2}\right)^{2}} \log \left(1+\frac{p^{2}}{m^{2}}\right)\right)\right. \\
& +i p_{\mu}\left(2 \alpha \frac{m}{p^{2}}+6 \frac{m}{p^{2}}-\left(2 \alpha \frac{m^{3}}{\left(p^{2}\right)^{2}}+6 \frac{m^{3}}{\left(p^{2}\right)^{2}}\right) \log \left(1+\frac{p^{2}}{m^{2}}\right)\right) \\
& \left.-\not p p_{\mu}\left(2 \alpha \frac{1}{p^{2}}-4 \alpha \frac{m^{2}}{\left(p^{2}\right)^{2}}+4 \alpha \frac{m^{4}}{\left(p^{2}\right)^{3}} \log \left(1+\frac{p^{2}}{m^{2}}\right)\right)\right] \\
& \Lambda_{T}^{1-\text { loop }}=\sigma_{\mu \nu}+\frac{g^{2} C_{F}}{16 \pi^{2}}\left[\gamma _ { \mu } \gamma _ { \nu } \left(e_{1}-\lambda_{T}+3.79201 \alpha-(1-\alpha)\left(2 \frac{m^{2}}{p^{2}}-\log \left(a^{2} m^{2}+a^{2} p^{2}\right)\right.\right.\right. \\
& \left.\left.-\left(2 \frac{m^{4}}{\left(p^{2}\right)^{2}}+\frac{m^{2}}{p^{2}}\right) \log \left(1+\frac{p^{2}}{m^{2}}\right)\right)\right) \\
& -\left(\gamma_{\mu} \not p p_{\nu}-\gamma_{\nu} \not p p_{\mu}\right)(1-\alpha)\left(\left(4 \frac{m^{4}}{\left(p^{2}\right)^{3}}+2 \frac{m^{2}}{\left(p^{2}\right)^{2}}\right) \log \left(1+\frac{p^{2}}{m^{2}}\right)-4 \frac{m^{2}}{\left(p^{2}\right)^{2}}\right) \\
& -i \gamma_{\mu} \gamma_{\nu} \not \supset\left(4 \frac{m^{3}}{\left(p^{2}\right)^{2}} \log \left(1+\frac{p^{2}}{m^{2}}\right)-4 \frac{m}{p^{2}}\right) \\
& \left.-i\left(\gamma_{\mu} p_{\nu}-\gamma_{\nu} p_{\mu}\right)\left(4 \frac{m}{p^{2}}-4 \frac{m^{3}}{\left(p^{2}\right)^{2}} \log \left(1+\frac{p^{2}}{m^{2}}\right)\right)\right] \\
& \Lambda_{A}^{1-\text { loop }}=\gamma_{5} \gamma_{\mu}+\frac{g^{2}}{16 \pi^{2}} C_{F} \gamma_{5}\left[\gamma _ { \mu } \left(e_{1}-\lambda_{A}+4.79021 \alpha-(2-\alpha) \frac{m^{2}}{p^{2}}-\alpha \log \left(a^{2} m^{2}+a^{2} p^{2}\right)\right.\right. \\
& \left.+\left(2(1-\alpha) \frac{m^{4}}{\left(p^{2}\right)^{2}}-2(1+\alpha) \frac{m^{2}}{p^{2}}\right) \log \left(1+\frac{p^{2}}{m^{2}}\right)\right) \\
& -i p_{\mu}(1-\alpha)\left(2 \frac{m}{p^{2}}-2 \frac{m^{3}}{\left(p^{2}\right)^{2}} \log \left(1+\frac{p^{2}}{m^{2}}\right)\right) \\
& +i \gamma_{\mu} \not \supset(1-\alpha)\left(2 \frac{m}{p^{2}}-2 \frac{m^{3}}{\left(p^{2}\right)^{2}} \log \left(1+\frac{p^{2}}{m^{2}}\right)\right) \\
& -\not p p_{\mu}\left(-8 \frac{m^{2}}{\left(p^{2}\right)^{2}}+2 \alpha \frac{1}{p^{2}}+4 \alpha \frac{m^{2}}{\left(p^{2}\right)^{2}}\right. \\
& \left.\left.+\left(8 \frac{m^{4}}{\left(p^{2}\right)^{3}}-4 \alpha \frac{m^{4}}{\left(p^{2}\right)^{3}}+4 \frac{m^{2}}{\left(p^{2}\right)^{2}}-4 \alpha \frac{m^{2}}{\left(p^{2}\right)^{2}}\right) \log \left(1+\frac{p^{2}}{m^{2}}\right)\right)\right] \\
& \Lambda_{P}^{1-\text { loop }}=\gamma_{5}+\frac{g^{2} C_{F}}{16 \pi^{2}} \gamma_{5}\left[e_{1}-\lambda_{P}+5.79201 \alpha-(\alpha+3) \frac{m^{2}}{p^{2}} \log \left(1+\frac{p^{2}}{m^{2}}\right)-(\alpha+3) \log \left(a^{2} m^{2}+a^{2} p^{2}\right)\right]
\end{aligned}
$$

The quantities $\lambda_{\mathcal{O}}$ are independent of the mass, gauge parameter, external momentum, and lattice spacing; they depend on the gluon action and the stout parameters. As discussed earlier, we have employed different parameters for the two smearing steps; in fact, we have also kept the parameters of the action's smearing procedure $\left(\omega_{A_{1}}\right.$, $\left.\omega_{A_{2}}\right)$ 
distinct from the parameters of the operator smearing $\left(\omega_{\mathcal{O}_{1}}, \omega_{\mathcal{O}_{2}}\right)$. For the tree-level Symanzik action and for general values of the stout parameters we obtained

$$
\begin{aligned}
& \lambda_{S}=-34.3217+389.210\left(\omega_{A_{1}}+\omega_{A_{2}}\right)-1403.65\left(\omega_{A_{1}}^{2}+\omega_{A_{2}}^{2}\right)-5614.59 \omega_{A_{1}} \omega_{A_{2}} \\
& +23395.4\left(\omega_{A_{1}}^{2} \omega_{A_{2}}+\omega_{A_{1}} \omega_{A_{2}}^{2}\right)-106814 \omega_{A_{1}}^{2} \omega_{A_{2}}^{2}, \\
& \lambda_{V}=86.7568\left[\left(\omega_{A_{1}}+\omega_{A_{2}}\right)-\left(\omega_{\mathcal{O}_{1}}+\omega_{\mathcal{O}_{2}}\right)\right]-337.383\left[\left(\omega_{A_{1}}^{2}+\omega_{A_{2}}^{2}\right)-\left(\omega_{\mathcal{O}_{1}}^{2}+\omega_{\mathcal{O}_{2}}^{2}\right)\right] \\
& -1349.53\left(\omega_{A_{1}} \omega_{A_{2}}-\omega_{\mathcal{O}_{1}} \omega_{\mathcal{O}_{2}}\right)+5950.81\left[\left(\omega_{A_{1}}^{2} \omega_{A_{2}}+\omega_{A_{1}} \omega_{A_{2}}^{2}\right)-\left(\omega_{\mathcal{O}_{1}}^{2} \omega_{\mathcal{O}_{2}}+\omega_{\mathcal{O}_{1}} \omega_{\mathcal{O}_{2}}^{2}\right)\right] \\
& -28627.2\left(\omega_{A_{1}}^{2} \omega_{A_{2}}^{2}-\omega_{\mathcal{O}_{1}}^{2} \omega_{\mathcal{O}_{2}}^{2},\right) \\
& \lambda_{T}=8.88342+116.579\left(\omega_{A_{1}}+\omega_{A_{2}}\right)-200.588\left(\omega_{\mathcal{O}_{1}}+\omega_{\mathcal{O}_{2}}\right)-531.759\left(\omega_{A_{1}}^{2}+\omega_{A_{2}}^{2}\right) \\
& +780.590\left(\omega_{\mathcal{O}_{1}}^{2}+\omega_{\mathcal{O}_{2}}^{2}\right)-2095.16 \omega_{A_{1}} \omega_{A_{2}}+3154.24 \omega_{\mathcal{O}_{1}} \omega_{\mathcal{O}_{2}} \\
& +31.8743\left(\omega_{A_{1}}+\omega_{A_{2}}\right)\left(\omega_{\mathcal{O}_{1}}+\omega_{\mathcal{O}_{2}}\right)+9877.233\left(\omega_{A_{1}}^{2} \omega_{A_{2}}+\omega_{A_{1}} \omega_{A_{2}}^{2}\right) \\
& -13993.1\left(\omega_{\mathcal{O}_{1}}^{2} \omega_{\mathcal{O}_{2}}+\omega_{\mathcal{O}_{1}} \omega_{\mathcal{O}_{2}}^{2}\right)-284.001\left(\left(\omega_{A_{1}}+\omega_{A_{2}}\right) \omega_{\mathcal{O}_{1}} \omega_{\mathcal{O}_{2}}\right. \\
& \left.+\omega_{A_{1}} \omega_{A_{2}}\left(\omega_{\mathcal{O}_{1}}+\omega_{\mathcal{O}_{2}}\right)\right)-48519.3 \omega_{A_{1}}^{2} \omega_{A_{2}}^{2} \\
& +68237.1 \omega_{\mathcal{O}_{1}}^{2} \omega_{\mathcal{O}_{2}}^{2}+2709.49 \omega_{A_{1}} \omega_{A_{2}} \omega_{\mathcal{O}_{1}} \omega_{\mathcal{O}_{2}}, \\
& \lambda_{A}=17.0363+117.584\left(\omega_{A_{1}}+\omega_{A_{2}}\right)-314.355\left(\omega_{\mathcal{O}_{1}}+\omega_{\mathcal{O}_{2}}\right)-518.419\left(\omega_{A_{1}}^{2}+\omega_{A_{2}}^{2}\right) \\
& +1223.79\left(\omega_{\mathcal{O}_{1}}^{2}+\omega_{\mathcal{O}_{2}}^{2}\right)-2041.80 \omega_{A_{1}} \omega_{A_{2}}+4927.06 \omega_{\mathcal{O}_{1}} \omega_{\mathcal{O}_{2}} \\
& +31.8758\left(\omega_{A_{1}}+\omega_{A_{2}}\right)\left(\omega_{\mathcal{O}_{1}}+\omega_{\mathcal{O}_{2}}\right)+9559.98\left(\omega_{A_{1}}^{2} \omega_{A_{2}}+\omega_{A_{1}} \omega_{A_{2}}^{2}\right) \\
& -21823.5\left(\omega_{\mathcal{O}_{1}}^{2} \omega_{\mathcal{O}_{2}}+\omega_{\mathcal{O}_{1}} \omega_{\mathcal{O}_{2}}^{2}\right)-210.274\left(\left(\omega_{A_{1}}+\omega_{A_{2}}\right) \omega_{\mathcal{O}_{1}} \omega_{\mathcal{O}_{2}}\right. \\
& \left.+\omega_{A_{1}} \omega_{A_{2}}\left(\omega_{\mathcal{O}_{1}}+\omega_{\mathcal{O}_{2}}\right)\right)-47154.2 \omega_{A_{1}}^{2} \omega_{A_{2}}^{2} \\
& +105754 . \omega_{\mathcal{O}_{1}}^{2} \omega_{\mathcal{O}_{2}}^{2}+1396.94 \omega_{A_{1}} \omega_{A_{2}} \omega_{\mathcal{O}_{1}} \omega_{\mathcal{O}_{2}} \text {, } \\
& \lambda_{P}=25.7425+119.062\left(\omega_{A_{1}}+\omega_{A_{2}}\right)-428.120\left(\omega_{\mathcal{O}_{1}}+\omega_{\mathcal{O}_{2}}\right)-518.541\left(\omega_{A_{1}}^{2}+\omega_{A_{2}}^{2}\right) \\
& +1667.00\left(\omega_{\mathcal{O}_{1}}^{2}+\omega_{\mathcal{O}_{2}}^{2}\right)-2042.29 \omega_{A_{1}} \omega_{A_{2}}+6699.88 \omega_{\mathcal{O}_{1}} \omega_{\mathcal{O}_{2}} \\
& +31.8765\left(\omega_{A_{1}}+\omega_{A_{2}}\right)\left(\omega_{\mathcal{O}_{1}}+\omega_{\mathcal{O}_{2}}\right)+9435.40\left(\omega_{A_{1}}^{2} \omega_{A_{2}}+\omega_{A_{1}} \omega_{A_{2}}^{2}\right) \\
& -29654.0\left(\omega_{\mathcal{O}_{1}}^{2} \omega_{\mathcal{O}_{2}}+\omega_{\mathcal{O}_{1}} \omega_{\mathcal{O}_{2}}^{2}\right)-210.274\left(\left(\omega_{A_{1}}+\omega_{A_{2}}\right) \omega_{\mathcal{O}_{1}} \omega_{\mathcal{O}_{2}}\right. \\
& \left.+\omega_{A_{1}} \omega_{A_{2}}\left(\omega_{\mathcal{O}_{1}}+\omega_{\mathcal{O}_{2}}\right)\right)-44803.9 \omega_{A_{1}}^{2} \omega_{A_{2}}^{2} \\
& +143482 . \omega_{\mathcal{O}_{1}}^{2} \omega_{\mathcal{O}_{2}}^{2}+1657.76 \omega_{A_{1}} \omega_{A_{2}} \omega_{\mathcal{O}_{1}} \omega_{\mathcal{O}_{2}}
\end{aligned}
$$

We note in passing that in the absence of stout smearing $\left(\omega_{A_{i}}=\omega_{\mathcal{O}_{i}}=0\right) \lambda_{V}=0$, which implies that $Z_{\mathrm{V}}^{\mathrm{RI}}=Z_{\mathrm{V}}^{\overline{\mathrm{MS}}}=1$ [cf. Eqs. (59), (68)], as is well known from current conservation. In addition, Eq. (44) shows that nonrenormalization of $\mathcal{O}_{V}$ applies also when $\omega_{A_{i}}=\omega_{\mathcal{O}_{i}}$; this follows from the fact that the stout link version of $\mathcal{O}_{V}$ mimics that of the action, and thus current conservation applies equally well in this case.

The dependence of the Green's functions of Eqs. (38) - (42) on mass and external momentum is regularization independent and agrees for instance with the results of Refs. [22, 24]. As is well known, in the limit of zero mass the vector and axial Green's functions beyond tree level are not multiples of their tree-level values: There appear additional, finite contributions with tensor structures which are distinct from those at tree level. These contributions denoted as $\Sigma_{V}^{(2)}$ and $\Sigma_{A}^{(2)}$ can be read off Eqs. (39) and (41):

$$
\begin{aligned}
& \Sigma_{V}^{(2)}=\frac{g^{2} C_{F}}{16 \pi^{2}}\left[-2 \alpha \frac{q q_{\mu}}{q^{2}}\right] \\
& \Sigma_{A}^{(2)}=\frac{g^{2} C_{F}}{16 \pi^{2}}\left[-2 \alpha \frac{\gamma_{5} q q_{\mu}}{q^{2}}\right]
\end{aligned}
$$


A similar contribution for the tensor bilinear does not appear up to, and including, three loops [28]. The role of $\Sigma_{V}^{(2)}$ and $\Sigma_{A}^{(2)}$ in the renormalization of $\mathcal{O}_{V}$ and $\mathcal{O}_{A}$ will be discussed in the next section.

\section{RENORMALIZATION FUNCTIONS}

\section{A. Fermion field and fermion bilinear renormalization functions in the $\mathbf{R I}^{\prime}-\mathrm{MOM}$ scheme}

RFs for operators and action parameters relate bare quantities regularized on the lattice, to their renormalized continuum counterparts:

$$
\psi^{\text {renorm }}=Z_{\mathrm{q}}^{\frac{1}{2}} \psi^{\text {bare }}, \quad m^{\text {renorm }}=Z_{\mathrm{m}} m^{\text {bare }}, \quad \mathcal{O}_{\Gamma}^{\text {renorm }}=Z_{\mathcal{O}_{\Gamma}} \mathcal{O}_{\Gamma}^{\text {bare }}
$$

The RFs of lattice operators are necessary ingredients in the prediction of physical probability amplitudes from lattice matrix elements. In this section we present the multiplicative RFs in the RI'-MOM scheme of the fermion field $\left(Z_{\mathrm{q}}\right)$, the fermion mass $\left(Z_{\mathrm{m}}\right)$, and the fermion bilinears.

The RI'-MOM renormalization scheme consists in requiring that the forward amputated Green's function $\Lambda(p)$ computed in the chiral limit and at a given (large Euclidean) scale $p^{2}=\mu^{2}$ be equal to its tree-level value. Our results for the RFs are presented for arbitrary values of the renormalization scale $\mu$. This requirement leads to the following definitions for $Z_{\mathrm{q}}^{\mathrm{RI}^{\prime}}, Z_{\mathrm{m}}^{\mathrm{RI}}, Z_{\mathcal{O}_{\Gamma}}^{\mathrm{RI}^{\prime}}$ :

$$
\begin{aligned}
&\left.S_{1-\text { loop }}^{-1}\right|_{p^{2}=\mu^{2}, m=0}=\left.S_{\text {tree }}^{-1}\right|_{p^{2}=\mu^{2}, m=0} Z_{\mathrm{q}}^{\mathrm{RI}^{\prime}}(\mu), \\
&\left.\Sigma_{m}\right|_{p^{2}=\mu^{2}, m=0}=Z_{\mathrm{m}}^{\mathrm{RI}^{\prime}}(\mu) Z_{\mathrm{q}}^{\mathrm{RI}^{\prime}}(\mu), \\
&\left.\Lambda_{\mathcal{O}_{\Gamma}}^{1-\text { loop }}\right|_{p^{2}=\mu^{2}, m=0}=\Lambda_{\mathcal{O}_{\Gamma}}^{\text {tree }} Z_{\mathrm{q}}^{\mathrm{RI}^{\prime}}(\mu)\left(Z_{\mathcal{O}_{\Gamma}}^{\mathrm{RI}^{\prime}}(\mu)\right)^{-1}, \quad(\Gamma=S, T, P),
\end{aligned}
$$

where $S_{\text {tree }}^{-1}$ is the tree-level result for the inverse propagator, and $\Lambda_{\mathcal{O}_{\Gamma}}^{\text {tree }}$ is the tree-level value of the Green's function for $\mathcal{O}_{\Gamma}$.

The presence of $\Sigma_{V}^{(2)}$ and $\Sigma_{A}^{(2)}$ in the one-loop Green's functions of $\mathcal{O}_{V}$ and $\mathcal{O}_{A}$ makes a prescription such as Eq. (53) inapplicable in those cases. Instead we employ

$$
\left.\left(\Lambda_{V, A}^{1-\text { loop }}-\Sigma_{V, A}^{(2)}\right)\right|_{p^{2}=\mu^{2}, m=0}=\Lambda_{V, A}^{\text {tree }} Z_{\mathrm{q}}^{\mathrm{RI}^{\prime}}(\mu)\left(Z_{V, A}^{\mathrm{RI}{ }^{\prime}}(\mu)\right)^{-1}
$$

and thus take into account only the terms in $\Lambda_{V, A}$ which are proportional to their corresponding tree-level values.

The expressions we obtain using our results for $\Lambda_{\mathcal{O}_{\Gamma}}^{1-l o o p}$ are shown here only for the tree-level improved Symanzik gauge action. The quantities $\lambda_{\mathcal{O}}$ are defined in Eqs. (43) - (47). We note that the results for $Z_{\mathrm{m}}$ and $Z_{\mathrm{S}}$ are related by $Z_{\mathrm{m}}=Z_{\mathrm{S}}^{-1}$ as expected,

$$
\begin{gathered}
Z_{\mathrm{q}}^{\mathrm{RI}}=1+\frac{g^{2} C_{F}}{16 \pi^{2}}\left[-\alpha \log \left(a^{2} \mu^{2}\right)+4.79201 \alpha-7.21363+124.515\left(\omega_{A_{1}}+\omega_{A_{2}}\right)-518.433\left(\omega_{A_{1}}^{2}+\omega_{A_{2}}^{2}\right)\right. \\
\left.-2073.73 \omega_{A_{1}} \omega_{A_{2}}+9435.35\left(\omega_{A_{1}}^{2} \omega_{A_{2}}+\omega_{A_{1}} \omega_{A_{2}}^{2}\right)-45903.1 \omega_{A_{1}}^{2} \omega_{A_{2}}^{2}\right] \\
Z_{\mathrm{m}}^{\mathrm{RI}^{\prime}}=1+\frac{g^{2} C_{F}}{16 \pi^{2}}\left[-3 \log \left(a^{2} \mu^{2}\right)+\alpha+34.3217-389.210\left(\omega_{A_{1}}+\omega_{A_{2}}\right)+1403.65\left(\omega_{A_{1}}^{2}+\omega_{A_{2}}^{2}\right)\right. \\
\left.+5614.59 \omega_{A_{1}} \omega_{A_{2}}-23395.4\left(\omega_{A_{1}}^{2} \omega_{A_{2}}+\omega_{A_{1}} \omega_{A_{2}}^{2}\right)+106813 . \omega_{A_{1}}^{2} \omega_{A_{2}}^{2}\right]
\end{gathered}
$$




$$
\begin{aligned}
& Z_{\mathrm{S}}^{\mathrm{RI}^{\prime}}=1+\frac{g^{2} C_{F}}{16 \pi^{2}}\left[\lambda_{S}-\alpha+3 \log \left(a^{2} \mu^{2}\right)\right], \\
& Z_{\mathrm{V}}^{\mathrm{RI}^{\prime}}=1+\frac{g^{2} C_{F}}{16 \pi^{2}}\left[\lambda_{V}\right], \\
& Z_{\mathrm{T}}^{\mathrm{RI}^{\prime}}=1+\frac{g^{2} C_{F}}{16 \pi^{2}}\left[\lambda_{T}+\alpha-\log \left(a^{2} \mu^{2}\right)\right], \\
& Z_{\mathrm{A}}^{\mathrm{RI}^{\prime}}=1+\frac{g^{2} C_{F}}{16 \pi^{2}}\left[\lambda_{A}\right], \\
& Z_{\mathrm{P}}^{\mathrm{RI}^{\prime}}=1+\frac{g^{2} C_{F}}{16 \pi^{2}}\left[\lambda_{P}-\alpha+3 \log \left(a^{2} \mu^{2}\right)\right] .
\end{aligned}
$$

In order to compare perturbative and nonperturbative estimates of RFs one clearly needs to employ the same renormalization prescription in both cases. In the context of a numerical simulation the term $\Sigma^{(2)}$ for the vector and axial cases is often not removed from the Green's functions, in contrast to what is done perturbatively in Eq. (54). Therefore, an alternative RI' renormalization prescription appears more natural:

$$
Z_{q}^{-1} Z_{V, A}^{\mathrm{RI}^{\prime} \text { alter }} \operatorname{Tr}\left[\Lambda_{V, A}^{1-\text { loop }} \Lambda_{V, A}^{\text {tree }}\right]=\operatorname{Tr}\left[\Lambda_{V, A}^{\text {tree }} \Lambda_{V, A}^{\text {tree }}\right] .
$$

Using the above prescription, the extracted $Z_{\mathrm{V}}^{\mathrm{RI}}$ alter and $Z_{\mathrm{A}}^{\mathrm{RI}}$ alter take the form

$$
\begin{aligned}
& Z_{\mathrm{V}}^{\mathrm{RI}^{\prime} \text { alter }}=Z_{\mathrm{V}}^{\mathrm{RI}^{\prime}}+\frac{g^{2} C_{F}}{16 \pi^{2}} \frac{\alpha}{2}, \\
& Z_{\mathrm{A}}^{\mathrm{RI}^{\prime} \text { alter }}=Z_{\mathrm{A}}^{\mathrm{RI}^{\prime}}+\frac{g^{2} C_{F}}{16 \pi^{2}} \frac{\alpha}{2} .
\end{aligned}
$$

\section{B. Conversion to the $\overline{\mathrm{MS}}$ scheme}

In this section we provide the expressions for the RFs in the $\overline{\mathrm{MS}}$ continuum scheme using conversion factors adapted from Ref. [28]. These conversion factors do not depend on the regularization scheme (and, thus, they are independent of the lattice discretization) when expressed in terms of the renormalized coupling constant. However, expressing them in terms of the bare coupling constant introduces a dependence on the action. In our analysis we use one-loop formulas, which are action independent. The definition for the conversion factors $C_{\mathcal{O}}$ is as follows:

$$
Z_{\mathcal{O}_{\Gamma}}^{\overline{\mathrm{MS}}, \mathrm{NDR}}=C_{\mathcal{O}} Z_{\mathcal{O}_{\Gamma}}^{\mathrm{RI}^{\prime}}
$$


The above conversion factors refer to the naive dimensional regularization (NDR) of the $\overline{\mathrm{MS}}$ scheme (see e.g., Ref. [29]), in which $C_{P}=C_{S}$ and $C_{A}=C_{V}$. From Eq. (65) one obtains ${ }^{2}$

$$
\begin{aligned}
Z_{\mathrm{q}}^{\overline{\mathrm{MS}}, \mathrm{NDR}} & =Z_{\mathrm{q}}^{\mathrm{RI}^{\prime}}-\frac{g^{2} C_{F}}{16 \pi^{2}} \alpha+\mathcal{O}\left(g^{4}\right), \\
Z_{\mathrm{S}, \mathrm{P}}^{\overline{\mathrm{MS}}, \mathrm{NDR}} & =Z_{\mathrm{S}, \mathrm{P}}^{\mathrm{RI}^{\prime}}+\frac{g^{2} C_{F}}{16 \pi^{2}}(4+\alpha)+\mathcal{O}\left(g^{4}\right), \\
Z_{\mathrm{V}, \mathrm{A}}^{\overline{\mathrm{MS}}, \mathrm{NDR}} & =Z_{\mathrm{V}, \mathrm{A}}^{\mathrm{RI}}, \\
Z_{\mathrm{T}}^{\overline{\mathrm{MS}}, \mathrm{NDR}} & =Z_{\mathrm{T}}^{\mathrm{RI}}-\frac{g^{2} C_{F}}{16 \pi^{2}} \alpha+\mathcal{O}\left(g^{4}\right) .
\end{aligned}
$$

Other modified minimal subtraction schemes are related to NDR via additional finite renormalization and affect the operators which include a $\gamma_{5}$, due to the nonunique generalization of $\gamma_{5}$ to $\mathrm{D}$ dimensions. Thus, the treatment of the pseudoscalar and axial operators in the $\overline{\mathrm{MS}}$ scheme requires special attention. The $\overline{\mathrm{MS}}$ renormalized pseudoscalar and axial operators, as defined in the scheme of 't Hooft and Veltman (HV) [30], involve extra finite factors, $Z_{5}^{P}, Z_{5}^{A}$, in addition to the conversion factors of Eqs. (67) and (68) [31]:

$$
\begin{aligned}
& Z_{5}^{P}=1-\frac{g^{2}}{16 \pi^{2}}\left(8 C_{F}\right) \\
& Z_{5}^{A}=1-\frac{g^{2}}{16 \pi^{2}}\left(4 C_{F}\right) .
\end{aligned}
$$

The relation between the NDR and the HV schemes is

$$
\begin{aligned}
Z_{\mathrm{P}}^{\overline{\mathrm{MS}}, \mathrm{HV}} & =Z_{\mathrm{P}}^{\overline{\mathrm{MS}}, \mathrm{NDR}} Z_{5}^{P}, \\
Z_{\mathrm{A}}^{\overline{\mathrm{MS}}, \mathrm{HV}} & =Z_{\mathrm{A}}^{\overline{\mathrm{MS}}, \mathrm{NDR}} Z_{5}^{A} .
\end{aligned}
$$

We would like to point out that although the expressions for $Z_{5}^{A}$ and $Z_{5}^{P}$ are, in general, different for flavor singlet and nonsinglet operators, at one-loop level they coincide.

Other variants of $\overline{\mathrm{MS}}$ include the DREZ and DRED schemes; the conversion from one scheme to another can be found in Sec. 4 of Ref. [13]. Our results for the fermion bilinears using the Wilson gauge action and without stout smearing converted in the DREZ scheme agree with the corresponding results of Ref. [13].

Having obtained $Z_{\mathcal{O}_{\Gamma}}^{X}$ in some renormalization scheme $\left[X=\left(\mathrm{RI}^{\prime}\right),\left(\mathrm{RI}^{\prime}\right.\right.$ alter $),(\overline{\mathrm{MS}}, \mathrm{NDR}),(\overline{\mathrm{MS}}, \mathrm{HV})$, etc.] the expression for the renormalized Green's functions in that scheme $\Lambda_{\mathcal{O}_{\Gamma}}^{\text {renorm,X }}(p, m)$ follow immediately:

$$
\Lambda_{\mathcal{O}_{\Gamma}}^{\text {renorm }, X}(p, m)=\Lambda_{\mathcal{O}_{\Gamma}}^{\text {bare, } X}(p, m)\left(Z_{q}^{X}\right)^{-1} Z_{\mathcal{O}_{\Gamma}}^{X}
$$

\section{SUMMARY}

In this paper we presented the calculation of the fermion propagator and the Green's functions for the ultralocal fermion bilinear operators: scalar, pseudoscalar, vector, axial and tensor. The computations were performed to one loop in lattice perturbation theory, using staggered fermions and Symanzik improved gluons parameterized by three independent "Symanzik" coefficients; explicit results are presented for some of the most commonly used actions in this family: Wilson, tree-level Symanzik, tadpole improved Lüscher-Weisz, Iwasaki, and DBW2.

The novelty in our calculations was the stout smearing of the links that we applied in both the fermion action and in the bilinear operators. More precisely, we use two steps of stout smearing with distinguishable parameters. To make our results as general as possible we also distinguished between the stout parameters appearing in the fermion action and in the bilinears.

Our expressions for the fermion propagator and the Green's functions of the bilinear operators exhibit a rather nontrivial dependence on the external momentum $(q)$ and the fermion mass $(m)$, and they are polynomial functions of the gauge parameter $(\alpha)$, stout parameters $\left(\omega_{A_{i}}, \omega_{\mathcal{O}_{i}}\right)$, and coupling constant $(g)$. The numerical coefficients

\footnotetext{
${ }^{2}$ Note that, at variance with Eq. (68), the conversion factors $C_{V, A}$ will not be equal to 1 if one uses, e.g., the "alternative" RI' renormalization scheme of Eq. (62).
} 
appearing in these expressions depend on the Symanzik parameters of the gluon action and were presented for the tree-level Symanzik improved gluon action; the most general expressions can be found in the electronic document in the form of a Mathematica input file, Staggered.m.

Using the aforementioned results we extract the renormalization function of the fermion field and those of the fermion bilinears in the $\mathrm{RI}^{\prime}-\mathrm{MOM}$ scheme and we provide the appropriate conversion factors to the $\overline{\mathrm{MS}}$ scheme; we pay particular attention to the operators which include a $\gamma_{5}$ in their definition. Moreover, for the case of the vector and axial operators we give an alternative prescription to obtain the renormalizations in the $\mathrm{RI}^{\prime}$ scheme.

There are several directions in which the present work could be extended

- A natural extension would be the computation of the Green's functions for operators including covariant derivatives, such as the one-derivative vector and axial operators ${ }^{3}: \bar{\psi} \gamma_{\{\mu} \overleftrightarrow{D}_{\nu\}} \psi, \bar{\psi} \gamma_{5} \gamma_{\{\mu} \overleftrightarrow{D}_{\nu\}} \psi$. The corresponding renormalization functions may be applied to the nonperturbative lattice evaluation of the momentum fraction of the nucleon, $\langle x\rangle_{q}$, and the moment of the polarized quark distribution of the nucleon, $\langle x\rangle_{\Delta q}$.

- A related further work using staggered fermions with stout improvement would be a computation of Green's functions for four-Fermi operators; a work in this direction can be found in Ref. [32].

- A possible improvement to the action may involve further iterations of stout smearing; such a procedure has been applied to clover [33] and Wilson fermions [34].

- It would be also interesting to calculate the Green's functions up to second order in the lattice spacing; such an extension would not only be useful to constructing improved versions of the operators but also to remove $\mathcal{O}\left(g^{2} a^{2}\right)$ contributions from the nonperturbative estimates of the renormalization functions. Similar computations have been performed recently with Wilson/clover/twisted mass fermions [17, 23, 35].

Acknowledgments: This work was partly supported by funding received from the Cyprus Re-

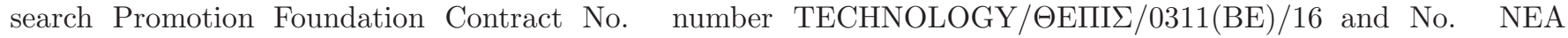
YПО $\Delta \mathrm{OMH} / \Sigma T$ PATH/0308/31. We acknowledge many helpful discussions with G. Bali, F. Bruckmann, and G. Endrődi.

\footnotetext{
${ }^{3}$ Curly brackets denote symmetrization and subtraction of the trace.
} 


\section{Appendix A: STOUT SMEARING OF THE LINKS}

Here we present the doubly stout one-gluon link, $U^{(1)}$, for general values of $\omega_{1}$ and $\omega_{2}$, as well as the two-gluon link, $U^{(2)}$ (only for $\omega_{2}=0$, to simplify the latter's lengthy expression):

$$
\begin{aligned}
\tilde{U}_{\mu}^{(1)}\left(x ; \omega_{1}, \omega_{2}\right)=i g[ & +A_{\mu}(x)+\left(\omega_{1}+\omega_{2}\right)\left(-8 A_{\mu}(x)+\sum_{\rho= \pm 1}^{ \pm 4}\left(A_{\mu}(x+a \hat{\rho})+A_{\rho}(x)-A_{\rho}(x+a \hat{\mu})\right)\right) \\
& +\left(\omega_{1} \omega_{2}\right)\left[64 A_{\mu}(x)+\sum_{\rho= \pm 1}^{ \pm 4}\left(-16 A_{\mu}(x+a \hat{\rho})-8 A_{\rho}(x)+8 A_{\rho}(x+a \hat{\mu})\right)\right. \\
& \left.\left.+\sum_{\rho= \pm 1}^{ \pm 4} \sum_{\sigma= \pm 1}^{ \pm 4}\left(A_{\rho}(x+a \hat{\sigma})-A_{\rho}(x+a \hat{\mu}+a \hat{\sigma})+A_{\mu}(x+a \hat{\rho}+a \hat{\sigma})\right)\right]\right], \\
\tilde{U}_{\mu}^{(2)}\left(x ; \omega_{1}, \omega_{2}=0\right)=g^{2}[ & -\frac{A_{\mu}(x)^{2}}{2}+\omega_{1}\left(8 A_{\mu}(x)^{2}-\sum_{\rho= \pm 1} A_{\mu}(x)\left(A_{\mu}(x+a \hat{\rho})+A_{\rho}(x)-A_{\rho}(x+a \hat{\mu})\right)\right) \\
& +\omega_{1}^{2}\left[-32 A_{\mu}(x)^{2}+\sum_{\rho= \pm 1}^{ \pm 4}\left(8 A_{\mu}(x)\left(A_{\mu}(x+a \hat{\rho})+A_{\rho}(x)-A_{\rho}(x+a \hat{\mu})\right)\right)\right. \\
& +\sum_{\rho= \pm 1}^{ \pm} \sum_{\sigma= \pm 1}^{ \pm 4}\left(-\frac{1}{2} A_{\mu}(x+a \hat{\rho}) A_{\mu}(x+a \hat{\sigma})-A_{\mu}(x+a \hat{\rho}) A_{\sigma}(x)\right. \\
& +\frac{1}{2} A_{\rho}(x) A_{\sigma}(x)+\frac{1}{2} A_{\rho}(x+a \hat{\mu}) A_{\sigma}(x)+\frac{1}{2} A_{\rho}(x+a \hat{\mu}-a \hat{\rho}) A_{\sigma}(x+a \hat{\mu}) \\
&
\end{aligned}
$$

where we define $A_{-\rho}(y)=-A_{\rho}(y-a \hat{\rho}), \rho>0$.

Note: The order in which a product of gluon fields appear in $\tilde{U}_{\mu}^{(2)}$ is irrelevant for the particular diagrams which we compute (since these two gluons are contracted among themselves); we have used this fact in order to simplify the expression for $\tilde{U}_{\mu}^{(2)}$. 


\section{Appendix B: NUMERICAL RESULTS FOR THE PROPAGATOR}

In this appendix we present the numerical coefficients $e_{1}$ and $e_{2}$ appearing in Eq. (36); these are polynomials in the two stout smearing parameters of the action $\left(\omega_{A_{1}}, \omega_{A_{2}}\right)$ :

$$
\begin{aligned}
e_{1} & =e^{(1,1)}+e^{(1,2)}\left(\omega_{A_{1}}+\omega_{A_{2}}\right)+e^{(1,3)}\left(\omega_{A_{1}}^{2}+\omega_{A_{2}}^{2}\right) \\
& +e^{(1,4)} \omega_{A_{1}} \omega_{A_{2}}+e^{(1,5)}\left(\omega_{A_{1}}^{2} \omega_{A_{2}}+\omega_{A_{1}} \omega_{A_{2}}^{2}\right)+e^{(1,6)} \omega_{A_{1}}^{2} \omega_{A_{2}}^{2}, \\
e_{2} & =e^{(2,1)}+e^{(2,2)}\left(\omega_{A_{1}}+\omega_{A_{2}}\right)+e^{(2,3)}\left(\omega_{A_{1}}^{2}+\omega_{A_{2}}^{2}\right) \\
& +e^{(2,4)} \omega_{A_{1}} \omega_{A_{2}}+e^{(2,5)}\left(\omega_{A_{1}}^{2} \omega_{A_{2}}+\omega_{A_{1}} \omega_{A_{2}}^{2}\right)+e^{(2,6)} \omega_{A_{1}}^{2} \omega_{A_{2}}^{2}
\end{aligned}
$$

The dependence of the quantities $e^{(i, j)}$ on the Symanzik coefficients cannot be given in closed form; their values for the Wilson and tree-level Symanzik gluon actions can be read in Table I. For other actions the values of $e^{(i, j)}$ are provided in electronic form (see Appendix C).

\begin{tabular}{ccc}
\hline \hline$e^{(i, j)}$ & Wilson & tree-level Symanzik \\
\hline \hline$e^{(1,1)}$ & -9.83170 & -7.21363 \\
$e^{(1,2)}$ & 167.367 & 124.515 \\
$e^{(1,3)}$ & -710.612 & -518.433 \\
$e^{(1,4)}$ & -2842.45 & -2073.73 \\
$e^{(1,5)}$ & 13134.2 & 9435.35 \\
$e^{(1,6)}$ & -64757.6 & -45903.1 \\
$e^{(2,1)}$ & 33.3933 & 27.1081 \\
$e^{(2,2)}$ & -342.525 & -264.695 \\
$e^{(2,3)}$ & 1174.37 & 885.215 \\
$e^{(2,4)}$ & 4697.49 & 3540.86 \\
$e^{(2,5)}$ & -18790.0 & -13960.0 \\
$e^{(2,6)}$ & 82920.9 & 60910.8 \\
\hline \hline
\end{tabular}

TABLE I: Numerical coefficients $e^{(i, j)}$ of the propagator for the Wilson and tree-level Symanzik actions. 


\section{Appendix C: NOTATION IN MATHEMATICA FILE STAGGERED.M}

The full body of our results can be accessed online through the file Staggered.m, which is a Mathematica input file [?]. ???In this distribution package we include a collection of our results in order to make them easily accessible to the reader. More details and definitions can be found in Appendix $\mathrm{C}$ of the paper. It includes the following.

\section{One- and two-gluon doubly stout links}

The expressions for the one- and two-gluon "doubly stout" links for different stout parameters in the first and second smearing step:

$$
\begin{aligned}
& \tilde{\tilde{U}}_{\mu}^{(1)}\left(x ; \omega_{1}, \omega_{2}\right)=U 1[x, \text { mu, omega } 1, \text { omega } 2] \\
& \tilde{\tilde{U}}_{\mu}^{(2)}\left(x ; \omega_{1}, \omega_{2}\right)=U 2[x, \text { mu, omega } 1, \text { omega } 2]
\end{aligned}
$$

The arguments of $\mathrm{U} 1$ and $\mathrm{U} 2$ are the following:

- $x$ : position of the link in the lattice

- mu: direction of the link

- omega1: the first stout parameter

- omega2: the second stout parameter

Moreover, the gluon field is denoted $\operatorname{as}^{4} A_{\sigma}(x+a \hat{\tau}) \equiv \mathrm{A}[\operatorname{sigma}, \mathrm{x}+$ tau $]$. The indices rho[1]-rho[4] appearing in $\tilde{\tilde{U}}_{\mu}^{(1)}$ and $\tilde{\tilde{U}}_{\mu}^{(2)}$ are dummy: a summation $\sum_{\text {rho[1]=+1 }}^{+4}$ is implied, but only in terms which contain rho[1]; similarly for rho[2]-rho[4].

\section{One-loop inverse propagator}

The one-loop inverse propagator is

$$
S_{1-\text { loop }}^{-1}=\text { propagator[Action, alpha, omegaA1, omegaA2, g2tilde, m] . }
$$

This expression depends on the following variables.

- Action (selection of improved gauge action as follows [24]:
1: Plaquette,
2: Tree Level Symanzik,
3: $\operatorname{TILW}\left(\beta c_{0}=8.60\right)$,
4: $\operatorname{TILW}\left(\beta c_{0}=8.45\right)$,
5: $\operatorname{TILW}\left(\beta c_{0}=8.30\right)$,
6: $\operatorname{TILW}\left(\beta c_{0}=8.20\right)$,
7: $\operatorname{TILW}\left(\beta c_{0}=8.10\right)$,
8: $\operatorname{TILW}\left(\beta c_{0}=8.00\right)$,

\footnotetext{
${ }^{4}$ Regarding the ordering in products of gluon fields in $\tilde{\tilde{U}}_{\mu}^{(2)}$ see Note in Appendix A.
} 
9: Iwasaki,

10: DBW2,

where $\beta=2 N_{c} / g_{0}^{2}$.

- alpha: gauge parameter (Landau/Feynman/generic correspond to 0/1/alpha)

- omegaA1(omegaA2): the first (second) stout parameter coming from the smearing of the links appearing in the action

- g2tilde: $\tilde{g}^{2} \equiv \frac{g^{2} C_{F}}{16 \pi^{2}} \quad$ (g: coupling constant)

- m: Lagrangian mass

- pslash $\equiv \not \supset$

- $\mathrm{p} 2 \equiv p^{2}$

\section{Amputated Green's functions}

The amputated Green's functions relevant to the ultralocal operators:

$$
\begin{aligned}
& \Lambda_{S}^{1-\text { loop }}=\quad \text { scalar[Action, alpha, omegaA1, omegaA2, omegaO1, omegaO2, g2tilde, m] } \\
& \Lambda_{P}^{1-\text { loop }}=\text { pseudoscalar[Action, alpha, omegaA1, omegaA2, omegaO1, omegaO2, g2tilde, m] } \\
& \Lambda_{V}^{1-\text { loop }}=\quad \text { vector[Action, alpha, omegaA1, omegaA2, omegaO1, omegaO2, g2tilde, m] } \\
& \Lambda_{A}^{1-\text { loop }}=\quad \text { axial[Action, alpha, omegaA1, omegaA2, omegaO1, omegaO2, g2tilde, m] } \\
& \Lambda_{T}^{1-\text { loop }}=\quad \text { tensor[Action, alpha, omegaA1, omegaA2, omegaO1, omegaO2, g2tilde, m] . }
\end{aligned}
$$

where

- omegaO1(omegaO2): the first (second) stout parameter coming from the smearing of the links appearing in the bilinears

- $\mathrm{p}[\mathrm{mu}] \equiv p_{\mu}$

- $\mathrm{p}[\mathrm{nu}] \equiv p_{\nu}$

- gamma5 $\equiv \gamma_{5}$

- $\operatorname{gamma1} \equiv \gamma_{\mu}$

- $\operatorname{gamma} 2 \equiv \gamma_{\nu}$

- gamma5gamma1 $\equiv \gamma_{5} \gamma_{\mu}$

- gamma5pslash $\equiv \gamma_{5} \not \supset$

- gamma5gamma1pslash $\equiv \gamma_{5} \gamma_{\mu} \not p$

- gamma1gamma $2 \equiv \gamma_{\mu} \gamma_{\nu}$

- gamma2gamma1 $\equiv \gamma_{\nu} \gamma_{\mu}$

- gamma1gamma2pslash $\equiv \gamma_{\mu} \gamma_{\nu} \not \supset$

- gamma1pslash $\equiv \gamma_{\mu} \not \supset$

- $\operatorname{gamma2pslash} \equiv \gamma_{\nu} \not \supset$

We note that Eqs. (C4) - (C8) hold for fermions with the same Lagrangian mass. 


\section{RFs}

The RFs of the fermion field and fermion bilinears in the RI'-MOM scheme:

$$
\begin{aligned}
Z_{\mathrm{q}}^{\mathrm{RI}} & =\mathrm{zq}[\text { Action, alpha, omegaA1, omegaA2, g2tilde, } \mathrm{p} 2] \\
Z_{\mathrm{S}}^{\mathrm{RI}} & =\mathrm{zs}[\text { Action, alpha, omegaA1, omegaA2, omegaO1, omegaO2, g2tilde, p2] } \\
Z_{\mathrm{P}}^{\mathrm{RI}} & =\mathrm{zp}[\text { Action, alpha, omegaA1, omegaA2, omegaO1, omegaO2, g2tilde, p2] } \\
Z_{\mathrm{V}}^{\mathrm{RI}} & =\mathrm{zv}[\text { Action, alpha, omegaA1, omegaA2, omegaO1, omegaO2, g2tilde, p2] } \\
Z_{\mathrm{A}}^{\mathrm{RI}} & =\text { za[Action, alpha, omegaA1, omegaA2, omegaO1, omegaO2, g2tilde, p2] } \\
Z_{\mathrm{T}}^{\mathrm{RI}} & =\mathrm{zt}[\text { Action, alpha, omegaA1, omegaA2, omegaO1, omegaO2, g2tilde, p2] }
\end{aligned}
$$

For convenience, all quantities in Eqs. (C3) - (C14) may be also retrieved using only their first argument; thus, for

example: vector[9] will result in $\Lambda_{V}^{1-\text { loop }}$ for the Iwasaki action with generic values of $\alpha, \omega_{A_{i}}, \omega_{\mathcal{O}_{i}}, g, m, p$.

[1] P. Hägler, Phys. Rept. 490, 49 (2010), [arXiv:0912.5483].

[2] T. Doi (HAL QCD Collaboration), PoS LATTICE2012, 009 (2012), [arXiv:1212.1572].

[3] H.-W. Lin, PoS LATTICE2012, 013 (2012), [arXiv:1212.6849].

[4] J. B. Kogut and L. Susskind, Phys. Rev. D11, 395 (1975).

[5] A. Bazavov, C. Bernard, C. DeTar, W. Freeman, S. Gottlieb, et al. (MILC Collaboration), Phys. Rev. D87, 054503 (2013), [arXiv:1212.4768].

[6] I. Allison, E. Dalgic, C. Davies, E. Follana, R. Horgan, et al. (HPQCD Collaboration), Phys. Rev. D78, 054513 (2008), [arXiv:0805.2999].

[7] G. Bali, F. Bruckmann, G. Endrődi, Z. Fodor, S. Katz, et al., JHEP 1202, 044 (2012), [arXiv:1111.4956].

[8] G. Bali, F. Bruckmann, M. Constantinou, M. Costa, G. Endrődi, et al., Phys. Rev. D86, 094512 (2012), [arXiv:1209.6015].

[9] Y. Aoki, Z. Fodor, S. D. Katz, and K. Szabo, JHEP 0601, 089 (2006), [hep-lat/0510084].

[10] S. Borsányi, Z. Fodor, S. Katz, S. Krieg, C. Ratti, et al. (Wuppertal-Budapest Collaboration), J. Phys. G38, 124060 (2011), [arXiv:1109.5030].

[11] A. Bazavov, T. Bhattacharya, M. Cheng, C. DeTar, H. Ding, et al., Phys. Rev. D85, 054503 (2012), [arXiv:1111.1710].

[12] S. Capitani, Phys. Rept. 382, 113 (2003), [hep-lat/0211036].

[13] A. Patel and S. R. Sharpe, Nucl. Phys. B395, 701 (1993), [hep-lat/9210039].

[14] S. Aoki, T. Izubuchi, Y. Kuramashi, and Y. Taniguchi, Phys. Rev. D67, 094502 (2003), [hep-lat/0206013].

[15] Q. Mason, H. D. Trottier, R. Horgan, C. T. Davies, and G. P. Lepage (HPQCD Collaboration), Phys. Rev. D73, 114501 (2006), [hep-ph/0511160].

[16] A. Skouroupathis and H. Panagopoulos, Phys. Rev. D76, 094514 (2007), [arXiv:0707.2906].

[17] C. Alexandrou, M. Constantinou, T. Korzec, H. Panagopoulos, and F. Stylianou, Phys. Rev. D83, 014503 (2011), [arXiv:1006.1920].

[18] W. Lee and S. R. Sharpe, Phys. Rev. D66, 114501 (2002), [hep-lat/0208018].

[19] J. Kim, W. Lee, and S. R. Sharpe, Phys. Rev. D81, 114503 (2010), [arXiv:1004.4039].

[20] C. Morningstar and M. J. Peardon, Phys. Rev. D69, 054501 (2004), [hep-lat/0311018].

[21] A. Skouroupathis and H. Panagopoulos, Phys. Rev. D79, 094508 (2009), [arXiv:0811.4264].

[22] S. Capitani, M. Göckeler, R. Horsley, H. Perlt, P. E. L. Rakow, et al., Nucl. Phys. B593, 183 (2001), [hep-lat/0007004].

[23] M. Constantinou, V. Lubicz, H. Panagopoulos, and F. Stylianou, JHEP 0910, 064 (2009), [arXiv:0907.0381].

[24] C. Alexandrou, M. Constantinou, T. Korzec, H. Panagopoulos, and F. Stylianou, Phys. Rev. D86, 014505 (2012), [arXiv:1201.5025].

[25] R. Horsley, H. Perlt, P. E. L. Rakow, G. Schierholz, and A. Schiller, Phys. Rev. D78, 054504 (2008), [arXiv:0807.0345].

[26] D. Daniel and S. N. Sheard, Nucl. Phys. B302, 471 (1988).

[27] N. Ishizuka and Y. Shizawa, Phys. Rev. D49, 3519 (1994), [hep-lat/9308008].

[28] J. Gracey, Nucl. Phys. B662, 247 (2003), [hep-ph/0304113].

[29] A. J. Buras and P. H. Weisz, Nucl. Phys. B333, 66 (1990).

[30] G. 't Hooft and M. Veltman, Nucl. Phys. B44, 189 (1972).

[31] S. Larin, Phys. Lett. B303, 113 (1993), [hep-ph/9302240, containing an extra section].

[32] J. Kim, W. Lee, and S. R. Sharpe, Phys. Rev. D83, 094503 (2011), [arXiv:1102.1774].

[33] S. Capitani, S. Durr, and C. Hoelbling, JHEP 0611, 028 (2006), [hep-lat/0607006].

[34] S. Borsányi, Z. Fodor, C. Hoelbling, S. D. Katz, S. Krieg, et al., PoS LATTICE2011, 209 (2011), [arXiv:1111.3500].

[35] M. Constantinou, M. Costa, M. Göckeler, R. Horsley, H. Panagopoulos, et al., Phys. Rev. D87, 096019 (2013), [arXiv:1303.6776]. 